\title{
LANGAGES ET PRATIQUES DU TIRAGE AU SORT DANS LA VIE PUBLIQUE DES COMMUNES MÉDIÉVALES ITALIENNES (XIII ${ }^{\mathrm{E}}-\mathrm{XIV}^{\mathrm{E}}$ SIËCLES)
}

Lorenzo Tanzini

De Boeck Supérieur | «Participations »

2019/0 Hors Série | pages 195 à 213

ISSN 2034-7650

ISBN 9782807393448

Article disponible en ligne à l'adresse :

https://www.cairn.info/revue-participations-2019-0-page-195.htm

Distribution électronique Cairn.info pour De Boeck Supérieur.

(c) De Boeck Supérieur. Tous droits réservés pour tous pays.

La reproduction ou représentation de cet article, notamment par photocopie, n'est autorisée que dans les limites des conditions générales d'utilisation du site ou, le cas échéant, des conditions générales de la licence souscrite par votre établissement. Toute autre reproduction ou représentation, en tout ou partie, sous quelque forme et de quelque manière que ce soit, est interdite sauf accord préalable et écrit de l'éditeur, en dehors des cas prévus par la législation en vigueur en France. Il est précisé que son stockage dans une base de données est également interdit. 


\section{Le monde médiéval et le monde moderne}




\title{
Langages et pratiques du tirage au sort dans la vie publique des communes médiévales italiennes (XIII ${ }^{\mathrm{e}} \mathrm{xIV}$ siècles)
}

\author{
> Lorenzo Tanzini
}

\section{> Résumé}

L'article porte sur la phénoménologie du tirage au sort dans les pratiques politiques des villes italiennes pendant la période « classique » de l'histoire urbaine du Moyen Âge. Dans ce contexte, le hasard n'est pas souvent utilisé en tant que tel, mais tout au contraire entre dans une composition complexe avec d'autres moyens de désignation, tels que le choix indirect ou la cooptation; l'emploi de chaque moyen se justifie en relation avec le moment politique, les changements de régime, les différents offices. Il s'agit donc de décoder les références documentaires pour comprendre le rôle effectif du tirage au sort et son arrière-plan idéologique, jusqu'au moment où, au cours du XIve siècle, le fonctionnement des systèmes électoraux dans la plupart des villes se fixe dans la forme de rotation des postes parmi les couches « qualifiées » de la société urbaine. 
'histoire médiévale, et surtout l'histoire des villes italiennes, apporte à l'étude des systèmes d'élection une série de questions et un langage qui sont nouveaux par rapport à la tradition classique'. D'ailleurs, l'étude des pratiques électorales dans les villes de l'Italie médiévale demande beaucoup de précautions dans l'usage des termes. Lorsque l'on parle d'élections au Moyen Âge, le mot recouvre de nombreux moyens de désignation, parmi lesquels l'élection proprement dite, mais aussi le tirage au sort (Théry, 2001). Il s'agit donc de comprendre le sens et les raisons de l'usage des différentes procédures. De plus, en approchant les mécanismes institutionnels des communes médiévales, il faut toujours expliquer les différents rôles politiques des offices, souvent cachés derrière l'usage des mêmes mots. L'élection du Podestat (potestas), le grand commis étranger qui vient dans la ville pour conduire la vie judiciaire et militaire pendant une année, a une fonction très différente de l'élection des Anziani qui constituent le gouvernement de la ville et l'expression de son leadership politique (Vigueur, Faini, 2010 ; Keller, 2014a) ; l'une et l'autre ne sont pas parfaitement comparables à l'élection des grands conseils de la ville, à laquelle la plupart des citoyens auront la possibilité de se présenter plusieurs fois dans leur vie, et qui permettent une participation de masse mais non un rôle d'initiative politique (Tanzini, 2014). Néanmoins, les statuts urbains et les pratiques institutionnelles parlent toujours d'élection : il y a donc la nécessité de distinguer au niveau des pratiques politiques ce qui paraît semblable au niveau de la norme.

Commençons par une célèbre discussion sur le tirage au sort dans la Florence du XIV siècle. Il s'agit d'une discussion à distance, entre le chroniqueur Giovanni Villani autour de 1340 et l'humaniste Leonardo Bruni, auteur de l'Histoire de Florence, au début du siècle suivant (lanziti, 2012). Tous les deux se penchent sur l'événement institutionnel fondamental qui a lieu en 1328, lorsque les autorités de Florence introduisent le système de recrutement des offices majeurs par Tratta (Guidi, 1972), c'est-à-dire par tirage au sort au sein d'une liste précédemment composée de citoyens.

Le marchand écrivain Villani décrit avec beaucoup de satisfaction le nouveau système de désignation, qui aurait pu assurer « un système [d'élection] bon et équitable » (« bello ordine e comune ») car il représentait une solution technique qui donnait à la commune « un fonctionnement régulier et pacifique («tranquillo e pacefico stato »), un véritable exemple pour les temps qui suivront » (Villani, 2001, p. 661). L'opinion de Villani est suivie par Marchionne di Coppo Stefani, qui jugeait le nouveau système un très bon moyen pour rendre le gouvernement de la ville «bon, franc et clair, et non pas factieux» («buono e franco e schietto e non settaiuolo »), pour éviter donc toute division sectaire (Marchionne di Coppo Stefani, 1903-1905, § 366). À l'opposé, Bruni déplorait le choix de 1328, inspiré par «quadam popularitate», disait-il, une «sorte de démocratie » dirait-on

[1] La version française a été révisée par Yves Sintomer. 
aujourd'hui : l'effet du tirage au sort est très mauvais parce que «la plupart de ceux que le sort désigne pour les offices sont des incapables » «indigni plerumque ad magistratum ex sortitione sumuntur ») par rapport à la tâche qui leur a été confiée (Bruni, 2001-2004).

À première vue, il s'agit d'une opposition classique, qui sera très forte quelques années après, notamment dans la réflexion de Guicciardini (Manin, 1995), entre la valeur « populaire » et participative du tirage au sort et les effets élitistes de l'élection - élitisme dont Leonardo Bruni est souvent considéré l'interprète dans la Florence du $x v^{e}$ siècle (Sintomer, 2009). Pourtant, la discussion introduit des éléments plus complexes: elle dépasse l'usage des mots, et implique le fonctionnement pratique du tirage au sort dans une tradition communale durable.

\section{Le tirage au sort, des origines à la maturité}

Au XIV siècle, le tirage au sort était déjà un système très ancien dans la vie publique des communes italiennes : il vaut donc la peine de suivre son histoire avant d'aborder les discussions théoriques sur le sujet. Dans la période des origines communales, puis pendant tout le $\mathrm{x}^{\mathrm{e}}{ }^{\mathrm{e}}$, nous ne trouvons pas de systèmes d'élection ad sortem, mais plutôt l'élection-cooptation des magistrats par le conseil restreint des consuls, souvent avec la variante (typiquement épiscopale) dite «par compromis » («per compromissum »), c'est-à-dire par l'élection directe d'un ou de quelques grands électeurs ${ }^{2}$ qui choisissent à leur tour d'autres collègues avec lesquels ils procéderont effectivement à l'élection pour pourvoir l'office en question (Keller, 2014a).

À Cremona par exemple, nous savons qu'en 1186, les consuls étaient élus «par compromis » («per viam compromissionis ») (Keller, 2014b). À Pistoia, l'ancien règlement déterminant l'élection des consuls (Breve dei Consoli, 1140-1180) prévoit que les consuls choisissent dans la grande assemblée du peuple (« in aringo ») deux citoyens chargés d'élire les 14 conseilleurs (Statuti di Pistoia, 1996, p. 173-175). En 1224, à Volterra, le grand électeur choisi par les consuls associe deux autres électeurs qu'il choisit pour former une sorte de collège électoral chargé de désigner tous ceux qui accéderont aux offices (Statuti di Volterra, 1951, p. 113). Ce mécanisme de choix à plusieurs échelons avait avant tout la fonction de dépolitiser l'élection, en remettant la véritable désignation à un cercle d'élus qui avaient la confiance de la communauté urbaine toute entière et qui ne pouvaient pas profiter de ce privilège pour leurs propres intérêts - puisque

[2] Dans les pages qui suivent, nous emploierons indifféremment les termes électeur ou grand électeur comme synonymes; cependant, seul le premier terme était employé à l'époque. L'usage du second vise seulement à faciliter la compréhension spontanée des lecteurs francophones contemporains. 
les grands électeurs n'étaient pas eux-mêmes éligibles aux offices qu'ils étaient chargés de pourvoir.

L'exemple de Volterra, qui date de 1224, est à cet égard très tardif, parce qu'au XIII ${ }^{\mathrm{e}}$ siècle, c'est le tirage qui tend à devenir presque partout la norme. À Reggio Emilia, le chapitre sur l'usage de ce système est le premier dans la compilation du droit communal (les Consuetudines) effectuée en 1242 : «Qu'aucun consul ou podestat ne soit élu sinon par tirage au sort » («Quod nullus eligatur in potestatem vel consulem nisi ad sortem ») (Consuetudini e statuti reggiani, 1933, p. 3-4). À Novare, en 1281, on prévoit que « les charges autant ordinaires qu'extraordinaires soient tirées au sort (« dentur et eligantur ad brevia ») (Statuta communitatis Novariae, 1876, coll. 541-544), et les statuts de Vérone prévoient presque la même chose en 1276 (Gli Statuti veronesi del 1276, p. 72-73).

Edoardo Ruffini, le plus important historien des systèmes électoraux de l'Italie communale, disait en 1927 que le tirage était « vieux comme le monde » (Ruffini, 1977), mais si l'on pose la question de sa généalogie dans les communes italiennes, la filiation précise du système est difficile à reconstruire. Personne ne croit plus, comme on le faisait encore dans les années de Ruffini, à une imitation consciente des pratiques des municipalités (municipia) romaines de l'antiquité par les communes médiévales; les études de Hagen Keller et de ses élèves portent plutôt sur les relations entre les pratiques électorales des communes et la tradition des élections épiscopales ou du Saint Empire romain germanique (Keller, 2014a). D'ailleurs, la tradition des systèmes électoraux dans le monde monastique a pu fournir plusieurs techniques aux pratiques en usage dans les villes (Moulin, 1953). Il est cependant vrai que les exemples religieux ou épiscopaux portent plutôt sur le modèle du vote per compromissionem, alors que le tirage au sort, au-delà de quelques exemples très rares, était expressément interdit dans l'Église, une bulle de Honorius III en 1225 ayant définitivement proscrit son usage dans ce cadre (Gaudemet, 1979, p. 328-331; Moulin, 1958). En tout état de cause, l'introduction du tirage au sort ne montre pas une filiation directe avec l'un ou l'autre des précédents historiques antiques et médiévaux. Sa diffusion a sans doute beaucoup à voir avec la complexification sociopolitique des communes au début du XIII ${ }^{e}$ siècle : puisque les conseils commençaient à inclure les citoyens de différentes couches sociales, il était nécessaire de choisir les officiers d'une manière plus complexe afin d'éviter les conflits.

Ce passage historique montre aussi la centralité des mécanismes de représentation dans les régimes communaux. Les institutions municipales du Moyen Âge n'étaient pas « représentatives » au sens moderne du mot, mais la nécessité d'une reproduction «descriptive »de la société par les institutions était très clairement perçue (Costa, 1999; La représentation politique, 2013). En raison de cette nécessité on trouve souvent dans les statuts urbains des normes sur le partage des différentes charges communales entre les différents quartiers de la ville, les catégories des personnes ou les couches sociales (Tanzini, 2018). 
C'est par cela que s'explique d'ailleurs probablement la différence très marquée entre les usages électoraux des villes italiennes et ceux des villes européennes du nord des Alpes, où l'on ne retrouve pas souvent le tirage au sort : à cette époque, l'homogénéité «bourgeoise » des élites municipales ne requérait pas le recours à un tel système, la cooptation étant le système le plus simple parce qu'il répondait à l'exigence de reproduction du groupe dirigeant (Rigaudière, 2002). La pluralité des groupes sociaux qui interviennent dans le jeu politique urbain au XIve siècle en Allemagne, avec la croissance des corporations (Zünften), y justifiait en effet parfois l'usage du tirage au sort, mais les cas restent assez limités (Isenmann, 1997 ; Richard, 2008, p. 200).

Les modalités particulières de la désignation ad sortem et de l'usage du tirage au sort n'avaient pas de connexion historique réelle avec les exemples antiques, grecs avant tout. Cependant, les premiers commentaires de la Politique d'Aristote, et notamment celui d'Albert le Grand (peu après 1260), qualifient le système de désignation par le klèrôtèrion comme la « raison du sort » (« ratio sortialis ») (Fioravanti, 1979 ; Cambiano, 2000, p. 372). Ce n'est pas un hasard que le commentaire d'Albert soit chronologiquement le plus proche de l'actualité institutionnelle des villes italiennes et en comporte le plus grand nombre de références, l'expression sortialis rappelant évidemment la désignation ad sortem en usage dans les villes de la même période. L'exemple antique n'était donc pas à l'origine des systèmes de désignation communaux, mais l'affinité technique avec la situation des cités classiques contribuait à consolider l'efficacité du système, ou à vérifier sa légitimité par rapport à la tradition. Un siècle plus tard, le Chancelier de la République florentine Coluccio Salutati, qui pouvait évidemment compter sur une connaissance beaucoup plus large des sources anciennes, pouvait désormais parler des systèmes électoraux de l'antiquité romaine (Baggioni, 2016, p. 302sq.).

Quoi qu'il en soit, au XIII ${ }^{e}$ siècle, la nécessité pratique de systèmes de désignation nouveaux croissante dans les villes italiennes l'emporte certainement sur une volonté d'imitation des Anciens, et on le constate même dans l'usage lexical. L'expression « electio ad sortem » - la plus classique - est fréquemment utilisée dans les sources de la période mais elle demeure ambigüe et non technique. Le mot «sors » peut même avoir le sens d' "expression de la volonté ou du choix dans le conseil »: à Reggio par exemple, dans les statuts de 1265, on discute «Sur le choix à faire dans le conseil utilisant les fèves blanches et noires » [ «De sorte danda in conscilio per fabas nigras et albas ») et dans ce texte, «dare sortem » veut dire tout simplement « voter », sans aucune référence au tirage au sort (Archivio di Stato di Reggio Emilia, Comune, Statuti 1, c. 67v-68r). Lorsqu'il s'agit de définir clairement cette dernière pratique, les textes utilisent davantage des locutions plus matérielles, telles que ad brevia, ad apodixas, ad polizzas, per rodolum : brevia ou apodixe étant les petits rouleaux employés pour le tirage des noms de ceux qui allaient occuper les offices.

La référence aux rouleaux présentait aussi l'avantage de ne pas évoquer l'image du hasard. En effet, celui-ci n’est pas explicitement considéré en tant que tel 
comme une valeur dans le langage politique des communes. On peut clairement l'apprécier dans les statuts communaux du XIII siècle, où les pratiques prévues pour le tirage au sort des officiers montrent une sorte de hasard indirect, corrigé. Cette correction vaut à plusieurs niveaux.

D'abord, même au cours de l'âge d'or du tirage au sort, les villes emploient des correctifs venus du système d'élection par compromis («per compromissum ») : à Viterbe, en 1251, on tire au sort deux noms parmi les membres du conseil, et ces deux électeurs choisissent à leur tour quatre autres personnes « intermédiaires » (« mediani ») avec lesquelles, réunis en commun avec les titulaires des charges déjà en place, ils vont élire les membres du nouveau conseil (Statuti della provincia romana, 1930, II, p. 99-100). Ici, la pratique de sélection rappelle donc les principes du vote par compromis (« compromissum »), mais avec un premier passage par le tirage au sort. L'une des versions les plus complexes lavec celle de Gênes) de cette désignation à plusieurs échelons est élaborée à Venise pour l'élection du Doge (Judde de Larivière, 2019).

En deuxième lieu - mais il s'agit de la caractéristique la plus importante des usages médiévaux du vote - le système ad brevia sert dans la plupart des villes à choisir les électeurs et non pas ceux qui occuperont les offices. La pratique est presque partout assez uniforme. Dans une grande assemblée, chacun prend un rouleau scellé : un petit nombre de rouleaux portent le symbole de l'inscription elector, et ceux qui trouvent l'inscription en ouvrant le rouleau seront les électeurs. Par conséquent, le hasard cesse au moment de l'élection finale : chaque électeur doit déclarer son choix. À Bologne, on conserve encore après 1264 (il s'agit sans doute là d'un cas unique) des fragments des listes des électeurs, chacun ayant inscrit le nom de l'élu de son choix. Il est vrai que parfois - à Bologne, à Lucques (Tamba, 1982 ; Statutum Lucani communis, 1991) - l'électeur peut se nommer soi-même, mais le plus fréquent est que l'électeur et l'élu ne doivent pas être la même personne. Le hasard du tirage au sort était donc strictement corrigé : puisque la pratique ad brevia était appliquée en groupes, souvent selon les quartiers de la population urbaine, l'électeur était exposé aux pressions de son milieu sociopolitique et familial. Par conséquent, le tirage au sort ne coupait pas les liens personnels qui conditionnaient le choix.

En troisième lieu, un autre facteur intervient pour limiter le hasard : ce ne sont pas tous les citoyens qui peuvent prendre le petit rouleau («qui possunt ire ad brevia »). Certes, on trouve parfois une forme extrêmement participative de cette modalité, par exemple à Padoue en 1271, où la rubrique XXI (« De electione offitialium ordinariorum ») des statuts reconnaît la possibilité d'être tiré pour l'élection des offices à tous les citoyens enregistrés dans les rouleaux fiscaux (Statuti del comune di Padova, 1873). Une situation similaire existe à Plaisance, où le statut de 1323 prévoit un conseil général (« consilium generalissimum ») ouvert à tout citoyen qui demande d'y participer (Statuto di Piacenza del 1323, 2012, p. 13). Mais le plus souvent, le tirage est fait in consilio, c'est-à-dire que seuls les membres du conseil peuvent prendre les brevia. À Pérouse, en 1279 , les noms de tous ceux 
qui occuperont les offices doivent être tirés dans le conseil au commencement de l'office du podestà ou du capitano: «Que tous les officiers de la commune de Pérouse soient élus par le Conseil majeur (per maius consilium civitatis et populi) par tirage au sort (sorte brevium), peu après l'entrée en charge du Podestat et du Capitaine du peuple » (Statuto di Perugia del 1279, 1996, p. 104-106). L'un des premiers devoirs du Podestat et du Capitaine du peuple sera donc de convoquer une séance du conseil pour effectuer le tirage au sort. Les membres des conseils communaux au $\mathrm{XII}^{\mathrm{e}}$ siècle sont extrêmement nombreux (plusieurs centaines de personnes même dans les villes de taille moyenne), mais l'admission au conseil demande pourtant des conditions relatives au patrimoine, au statut juridique, au métier : il s'agit donc d'un tirage au sort entre un nombre de personnes déjà présélectionnées.

Quatrième limitation : tandis que parfois le grand électeur choisit directement son élu, dans les pratiques suivies dans la plupart des villes (notamment pour les offices majeurs), il y a un passage intermédiaire et non une correspondance directe entre un électeur-un élu. Par exemple, en 1284 à Pistoia, douze électeurs sont tirés au sort pour chaque office majeur, et ils doivent choisir entre eux le nom de l'élu à une majorité des deux tiers (Breve et ordinamenta populi Pistorii, 2003, p. 86). À Padoue en 1277, sept électeurs sont tirés au sort, ils doivent choisir deux citoyens parmi « les meilleurs de leurs quartiers » («de melioribus sui quarterii »), dont seulement un sera tiré au sort : ici, tirage au sort et choix direct alternent deux fois pour la même élection (Statuti del comune di Padova, 1873). En passant, notons qu'il existe un exemple de cette procédure dans l'Écriture, dans le récit des Actes des Apôtres $(1,26)$ lorsque il s'agit de choisir le douzième apôtre après la mort de Judas : la communauté choisit deux fidèles, Matthias et Joseph

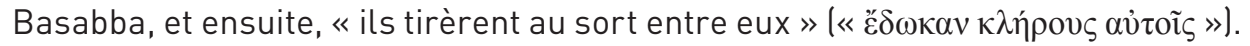

Le passage intermédiaire entre le tirage et l'élection a parfois lieu dans le Conseil. Un exemple très clair se trouve dans les statuts de Parme en 1266, qui montre le jeu de bascule entre hasard et élection à l'intérieur du système ad sortem-ad brevia, lorsqu'il s'agit de désigner au sein du Conseil les électeurs qui décideront des personnes qui occuperont les offices publics : après qu'un conseiller a été tiré au sort selon la procédure, il ne deviendra électeur que s'il recueille le vote de ses collègues présents ( «si vocatus per illam cedulam sortem habuerit, veniat ad faciendum electionem » (Statuta communis Parmae ab anno MCCLXVI, 1857, p. 39-40). Ici « habere sortem » veut dire en même temps « avoir la chance » d'être tiré au sort pour devenir électeur et « recevoir le bulletin de vote » des conseillers.

Plutôt que de poursuivre cette énumération, dégageons une première conclusion. Le tirage au sort au $\mathrm{XII}^{\mathrm{e}}$ siècle est clairement considéré comme le système par excellence pour l'élection aux offices. Mais en même temps, et en dépit de cette centralité, le tirage au sort n'a presque jamais la fonction d'assurer le hasard dans le choix de personnes qui occuperont les charges publiques. Sarah Blanshei, dans une remarquable étude du recrutement politique dans la ville de 
Bologne entre le XIII et XIV siècles, a bien montré que l'usage de différents mécanismes de tirage au sort pour l'élection des membres du conseil de la commune n'empêchait pas que celui-ci reproduise la composition politique du gouvernement, c'est-à-dire du collège des Anziani (Blanshei, 2010 ; Vallerani, 2011). On ne peut pas penser à un accident statistique : il s'agit plutôt d'un mécanisme structurel.

La véritable fonction du tirage est plutôt d'assurer que le choix des officiers soit bien réparti entre les différents groupes qui composent la société politique de la ville et, à cet égard, le tirage ad brevia semble la solution la plus naturelle. Il faut rappeler, en suivant les suggestions de l'école de Hagen Keller, que les élections au Moyen Âge ont avant tout une fonction de sauvegarde de la paix et de construction de consensus, plus que de confrontation d'alternatives politiques (Dartmann, 2012 ; Keller, 2014a). Cela vaut peut-être aussi pour l'Ancien régime tout entier (Christin, 2014). Yves Sintomer, dans son histoire du tirage au sort, a souligné que dans les grandes républiques médiévales, le tirage au sort a une fonction de consensus et de résolution des conflits, plus que de participation à l'instar de la démocratie grecque (Sintomer, 2011). Christoph Dartmann (2010) a parlé, peut-être avec un peu d'exagération, d'une "culture [politique] de la défaite», c'est-à-dire que le système électif devait avant tout assurer que la partie vaincue accepte la défaite sans entraîner une confrontation violente : l'intervention du tirage au sort, même seulement rituelle, s'expliquerait dans le cadre de cette culture. L'exagération réside sans doute dans l'omission des conflits farouches qui surgissent quand même à l'intérieur d'un tel système, et aussi bien souvent, du fait que la partie opposée au gouvernement de la ville était tout simplement écartée de la participation politique (Milani, 2003). Il faut donc porter l'attention à la définition sociopolitique du nombre de ceux qui peuvent réellement prendre part au rituel du tirage au sort. Néanmoins, il est évident que ce dernier répond d'abord à la nécessité de nuancer une reproduction brutale de l'hégémonie politique d'un parti dans les structures de la ville, parce que ce rituel efface l'expression directe de la volonté derrière ce que l'on pourrait appeler le voile du hasard.

Pour cette raison, les pratiques électorales des villes communales montrent une complexité rituelle presque religieuse : cette ritualité n'était pas un accessoire formel, elle représentait et conservait la force symbolique de la désignation. Le chroniqueur Marchionne rappelle quaprès la votation pour constituer les listes de citoyens entre lesquels il sera ultérieurement tiré au sort, les boîtes utilisées sont ouvertes dans un endroit interdit à ceux qui ont voté, choisi par six religieux : deux dominicains, deux franciscains et deux augustiniens, étrangers et non florentins; après l'ouverture des boîtes, les bourses (borse) contenant les noms qui seront tirés au sort doivent être gardées dans le chapitre de Sainte-Croix dans une armoire à trois clés, la première confiée au gardien des franciscains, la deuxième au prieur du monastère cistercien de Settimo et la dernière au capitaine du peuple (Marchionne di Coppo Stefani, 1903-1905, § 366). D'ailleurs, la sacralisation des procédures électorales est liée à l'usage de termes qui ont une évidente valeur religieuse : votum, electio, suffragium. Il vaut 
la peine ici de rappeler qu'en italien, votum signifie autant « vœu » que « vote »; l'« electio » par excellence est celle de Dieu choisissant son peuple, et le «suffrage » désigne normalement les personnes en l'honneur ou à la mémoire desquelles la messe est donnée lune messe en l'honneur - in suffraggio - des défunts est une expression commune en italien). Ceux qui participent à la procédure sont bien conscients de cette inclination religieuse du lexique institutionnel : à Sienne par exemple, en 1262, les rouleaux « signés » avec lesquels le conseiller devient électeur des officiers chargés d'amender le statut de la ville (emendatores constituti) portent l'inscription «A et $\Omega$ » (/l constituto del comune di Siena dell'anno 1262, 1896, p. 56-57), et impliquent donc une très forte association entre la tâche de l'électeur et une vocation divine ( «je suis l'alpha et l’oméga », Apocalypse I,8). Même si le rituel n'envisage pas la présence de symboles religieux, le déroulement de la procédure électorale est souvent présenté dans les statuts urbains avec une solennité exceptionnelle : à Foligno en 1350, le décompte des votes par le notaire du conseil utilise une boîte en cuivre, pour que le son de chaque boule qui tombe puisse être clairement entendu par tous les présents (Statuta communsi Fulginei, 1969, II, p. 30-32). On peut imaginer le silence attentif et tendu des conseilleurs pendant le décompte.

\section{Changements au XIV siècle}

Les systèmes « mixtes » de tirage au sort au XII" siècle que nous avons vus jusquelà avaient évidemment des défauts. Ils nécessitaient de fréquentes séances des conseils pour les brevia, c'est-à-dire pour le tirage au sort par rouleaux des officiers qui devaient intervenir peu après que le Podestat fût entré en charge, et impliquaient donc une procédure complexe qui gênait l'activité ordinaire ; surtout, ils n'effaçaient pas complètement l'expression de la volonté individuelle de l'électeur : ce point affaiblissait la fonction de consensus de l'élection «par rouleaux » (ad brevia).

Dès la fin du XIII siècle, et surtout au début du XIVe, les régimes communaux introduisent de nouveaux correctifs ou des changements radicaux dans le système. À Bologne, dès 1288, les statuts changent le mode d'élection des Anziani, les titulaires des offices majeurs de la ville (Statuti di Bologna dell'anno 1288, 1937, p. 297-298). Auparavant, ils étaient désignés par les corporations municipales: chacune tirait au sort parmi les membres de la corporation un certain nombre d'électeurs qui élisaient ensuite un Anziano qui « représentait » la corporation. Les statuts, se méfiant de la sagesse des associations corporatives, imposent une seconde étape : les corporations doivent proposer six élus, et le conseil a la tâche de choisir parmi eux ceux qui sont recrutés pour la charge d'Anziano. Le tirage au sort est donc limité par un choix, mais il s'agit d'un choix fait par l'assemblée et non par des grands électeurs. L'idée d'une élection directe des titulaires des offices est répandue dans l'Italie du début du XIVe siècle : dans les statuts de Pise, les membres du conseil sont choisis par les Anziani (Brevi del comune e del popolo, 1998, § I, 55), tandis qu'à Florence, le choix revient aux Prieurs 
(les premiers magistrats de la ville), épaulés par 40 《sages » (《sapientes ») (Statuti della Repubblica fiorentina, 1999, I, p. 32-33). D'une certaine manière, on peut observer qu'il s'agit d'une sorte de renouvellement de l'ancien système d'élection des consuls du XII ${ }^{\mathrm{e}}$ siècle - mais c'est ici le conseil des Anziani ou des Prieurs, les leaders du régime populaire, qui conduit cette forme très «politisée » de choix. Le sens du système réside dans l'idée que les collèges populaires des Anziani ou leurs conseils exercent une sorte de « représentation symbolique » de la communauté urbaine, et donc que leur choix a la valeur d'une élection par la communauté urbaine toute entière.

Une alternative pouvait être la création d'un conseil qui avait la tâche exclusive de pourvoir aux autres offices. Le tirage au sort ad brevia demeure donc, mais le nombre de ceux qui peuvent y participer est figé dans une assemblée ad hoc. Le système est là encore introduit à Bologne, mais pour les offices mineurs: les Conseils des 2000 ou des 4000 ne sont pas de véritables conseils, mais ils désignent plutôt le nombre de ceux qui peuvent «participer au tirage au sort » («ire ad brevia ») (Tamba, 1982). En 1306, à Modène, après une période de soumission seigneuriale à la famille d'Este, la commune retrouve sa liberté, et l'un des premiers actes est la constitution du conseil des 1600 (Modène est à cette époque une ville de 20000 habitants environ), qui sert non pour les délibérations ordinaires, mais «pour procéder à la désignation des officiers de la commune de Modène qui sont élus par tirage au sort («ad faciendum ellectiones officialium comunis Mutine que fient ad brevia »), et pour prononcer les condamnations et sentences » (Respublica mutinensis, 1929-1932). Un conseil vraiment «à la grecque », donc, consacré aux jugements publics et au tirage au sort des offices.

En tout état de cause, la tâche de ces conseils géants n'implique normalement pas l'élection des offices majeurs, tels que les Anziani, auxquels le gouvernement de la commune est confié. Dans les villes italiennes du XIII ${ }^{\mathrm{e}}$ ou XIV ${ }^{\mathrm{e}}$, il existe des centaines d'offices mineurs, qui sont liés à l'administration quotidienne ou au gouvernement des communautés rurales soumises: la désignation à cette gamme d'offices a une fonction centrale de reconnaissance du prestige et de la dignité sociale des citoyens qui peuvent être tirés au sort, mais elle demeure en-deçà du pouvoir politique réel. Par ailleurs, cette distinction entre la multiplicité des offices mineurs et la pleine responsabilité politique du cercle restreint du gouvernement empêche de considérer les régimes communaux comme des démocraties directes : même dans les formes plus participatives de désignation, le choix du noyau central du gouvernement garde un lien avec des modes de désignation plus complexes et sans doute moins « aveugles ».

À cet égard, la nouveauté la plus remarquable du XIve siècle vient des villes toscanes, notamment Sienne et Florence, où les changements des systèmes électoraux débouchent sur une pratique nouvelle, celle de la Tratta. Tratta - le mot vient de la tradition florentine - veut dire tout simplement «tirage au sort ». Mais le sens du mot est désormais très différent de celui qu'il avait à l'origine: encore un glissement dans l'acception du terme. Il s'agit de composer d'abord 
une liste des citoyens qui sont publiquement jugés dignes de recevoir la charge des offices. La composition de cette liste est remise à plusieurs collèges qui travaillent chacun de son côté, et dont les listes sont ensuite additionnées lphase de la recata - «apportée », «présentée »). L'étape suivante est décisive : la recata, c'est-à-dire la liste cumulée, est soumise à la votation, nom après nom, dans le grand conseil (à Sienne) ou dans un conseil ad hoc d'une centaine de citoyens choisis par le gouvernement là Florence). Les noms qui sont approuvés par la majorité sont introduits dans des bourses électorales, confiées aux différentes communautés religieuses urbaines : désormais le tirage au sort est effectué à la fin de mandat de chaque titulaire d'office, en tirant le nouveau nom de la bourse jusqu'à vider celle-ci au bout de quelques années (Najemy, 1982). Il est évident qu'ici, le hasard a presque disparu : il joue seulement un rôle quant à l'ordre dans lequel tous les noms introduits dans la bourse seront tirés pour l'office. Lorsqu'un citoyen a son nom introduit dans la bourse, il doit juste attendre son tour, sauf s'il perd le droit à l'office parce qu'il est absent, décédé ou parce qu'il n'a pas payé ses impôts. Pourtant, par une ironie de l'histoire, ce système sera dans les pratiques et dans la discussion politique de la fin du Moyen Âge l'image par excellence du tirage au sort-c'est justement sur ce système que joue la discussion entre Villani et Bruni. L'ironie était qu'il était la moins « aveugle » des formes de tirage au sort introduites pendant l'histoire communale.

Il faut bien comprendre ce passage marqué par l'introduction de la Tratta. Il s'agit d'une nouveauté essentielle pour le fonctionnement des techniques électorales, une solution effective aux défauts du système. Mais elle ne change pas la conception communale du tirage au sort en politique. Tout d'abord, elle maintient l'ensemble de l'appareil lexical et rituel du hasard (et on a vu qu'il s'agissait presque uniquement d'un appareil rituel) : le secret pendant le vote, l'interdiction de manifester ouvertement son choix pour ou contre les noms de la recata, l'impossibilité pour les citoyens d'accéder matériellement aux bourses et de connaître leur contenu une fois celles-ci remplies - bref, l'exclusion de toute volonté directe. La vie politique du début du XIV ${ }^{e}$ siècle - rappelons ici les paroles de Giovanni Villani citées au début de cet article sur la tratta - percevait en effet cette dernière comme un perfectionnement des systèmes classiques de tirage au sort, même si le nouveau mécanisme écartait en réalité tout hasard. Une fois encore, la culture politique communale ne considérait pas que le hasard était au centre du système de tirage au sort : le véritable centre du système résidait au contraire dans la rotation des offices entre ceux que la société urbaine percevait comme des citoyens politiquement qualifiés (Vallerani, 1994). Au XIII' siècle, cette reconnaissance venait de la participation au conseil loù se déroulait la procédure du tirage au sort par brevial, tandis que dans les grandes villes du xIV siècle, elle venait plutôt d'une qualification électorale par le biais de la recata, même si cela ne change pas vraiment les principes essentiels: chaque personne qui entre dans le cercle des qualifiés, que ce soit à travers la participation massive dans le conseil du xIII siècle ou parce qu'elle a obtenu la confiance des auteurs de la recata, est amenée à jouer son rôle actif de citoyen en tant que titulaire d'un office. Cela ne signifie pas, bien évidemment, que le tirage au sort 
soit une fiction inutile. La nécessité de dépolitiser le choix, de montrer que les titulaires des offices reproduisent en quelque sorte « naturellement » la société urbaine, donne au rituel de la désignation par tirage au sort un rôle réel. Il ne s'agit pas d'une nouveauté absolue : l'histoire du vote pendant l'antiquité, à Rome surtout, montre bien la centralité de la dimension rituelle de l'élection, même lorsque l'expression de la volonté reste presque totalement absente (Hollard, 2010, p. 231-233), et les systèmes d'élection au Moyen Âge ont par ailleurs généralement comme fonction première de mettre en scène l'unité et l'identité communautaire (Peneau, 2008, p. 27).

\section{La théorie}

On peut donc relire par ce prisme la « discussion à distance » entre Villani et Bruni. Lorsque celui-ci disait que la Tratta n'était pas un bon système, ce n'était pas seulement parce qu'elle risquait de confier des charges à des citoyens qui n'avaient pas de capacités politiques. Son argument est que la plupart de ceux qui sont tirés au sort s'avèrent incapables, parce que l'« on n'attend pas de la même manière les charges que l'on envisage aujourd'hui et celles qui viendront demain : au contraire, on voit très clairement et avec enthousiasme les premières, tandis que les futures paraissent distantes » « Neque enim pari diligentia providetur praesenti officio et multis secuturis; sed remota quidem illa, et an futura sint minime certa, hebetiori intuemur oculo »). Pour cette raison, être choisi dans une liste de noms parmi lesquels on sera tiré au sort dans le futur ne stimule pas l'attention que l'on porte à la charge en question, au contraire, elle l'affaiblit. Cela « éteint surtout la recherche de la vertu politique » («extinguit praeterea virtutis studium »). Au contraire, dans la compétition pour l'élection, les hommes publics se mettent en jeu de façon positive (« si suffragiis certandum foret...multo magis sese homines circumspicerent $\gg$ ).

La critique de Bruni porte directement sur le cœur du système, c'est-à-dire le principe selon lequel on est qualifié une seule fois et que l'on jouit par la suite de cette qualification dans les tirages au sort successifs. Selon Bruni, cette qualification définitive affaiblit l'attention portée à la fonction publique comme la recherche de la vertu (politique), parce que celui qui est certain d'avoir un office qui l'attend n'a plus rien à faire pour démontrer sa vertu : mieux vaudrait en conséquence suivre l'exemple des Romains et ouvrir chaque fois une véritable sélection entre candidats.

Il s'agit à mon avis d'une réflexion extrêmement profonde, qui dépassait peutêtre même la perception de l'élite florentine qui lisait et célébrait officiellement Bruni et son œuvre. Ici, on peut percevoir l'opposition entre deux modèles institutionnels et culturels de l'élection.

Dans la tradition communale, le mécanisme du tirage au sort est un système nécessaire mais en même temps complexe et difficile à gérer, qui souvent ne 
semble pas atteindre l'objectif essentiel que l'on attribue à l'élection. Les gouvernements communaux étaient conscients des défauts du système mais ils le gardaient pourtant parce que la ritualité du tirage au sort mettait en valeur les assemblées et les conseils, expressions de l'identité collective des citoyens. Dans une telle culture politique, les individus n'existent pas. Ce qui existe, ce sont les groupes, les corps, les collectivités urbaines où l'identité «publique » des individus est reconnue et admise à la représentation - les conseils, les corporations, les sociétés d'armes, les collèges qui composent la recata. Il s'agit d'un système très participatif, mais en même temps très fermé, parce qu'il n'envisage pas la possibilité d'expression pour ceux dont les groupes considèrent qu'ils ne font pas partie de «l'entre nous», qu'ils n'incluent pas dans le cercle « des nôtres ».

Tout au contraire, dans l'œuvre de Bruni, il y a l'idée que les individus doivent se confronter pour définir qui sont les meilleurs et les plus capables. Ce système sert avant tout à l'élite, parce que ce sont presque seulement les membres d'une élite qui peuvent montrer leurs qualités en termes d'expérience, de savoir-faire, de relations sociales; et en même temps, le système est plus ouvert que son parallèle communal, parce que l'individu a théoriquement toujours la possibilité de rejoindre les « autres », le cercle des meilleurs, quelle que soit son appartenance d'origine.

Ce n'est à mon avis pas un hasard si Bruni rappelle dans son argument l'exemple des Anciens : ici, le discours s'appuie sur l'image des élections, les suffragia de l'antiquité romaine, pour définir une idée nouvelle d'identité politique, opposée à la tradition communale récente. Ce n'est pas l'unique sujet au regard duquel la culture politique du $x v I^{e}$ siècle recourt au lexique de l'antiquité pour justifier un changement radical de ses valeurs politiques.

\section{Sources primaires}

Breve del comune e del popolo di Pisa del 1287, 1998, éd. par A. Ghignoli, Rome, ISIME.

Breve et ordinamenta populi Pistorii, a. 1284, 2003, éd. par Nelli R., Pinto G., Pistoia, Società storica pistoiese.

Bruni L., 2001-2004, Historiae Florentini populi, éd. par J. Hankins, 3 vol., Cambridge (Mass.), Harvard University Press.

Consuetudini e statuti reggiani del secolo XIII, 1933, éd. par Cerlini A., Milan, Hoepli. Il constituto del comune di Siena dell'anno 1262, 1896, éd. Zdekauer L., Milan, Hoepli.

Glistatuti veronesi del 1276 colle correzioni e le aggiunte fino al 1313 lcod. Campostrini, Bibl. Civica di Verona), I, 1940, éd. par Sandri G., Venise, Deputazione di storia patria per le Venezie.

Lo statuto di Piacenza del 1323, 2012, éd. par Fugazza E., Pavie, Pavia University press. 
Marchionne di Coppo Stefani, 1903-1905 , Cronaca fiorentina, éd. par Rodolico N., Città di Castello, Lapi.

Respublica Mutinensis (1306-1307), 1929-1932, éd. par Vicini E.P., II, Milan, Hoepli.

Statuta communis Fulginei, 1969, éd. par A. Messini, F. Baldaccini et alii, Perouse, Deputazione di storia patria per ll'Umbria.

Statuta communis Parmae ab anno MCCLXVI ad annum circiter MCCCIX (1857), éd. par Ronchini A., Parme, Fiaccadori.

Statuta communitatis Novariae, 1876, éd. Par Ceruti A., Turin Bocca, (HPM XVI, Leges Municipales, II).

Statuti del Comune di Padova dal secolo XII all'anno 1285, 1873, éd. par Gloria A., Padoue.

Statuti della provincia romana, I, 1910, éd. par Tomassetti F., Federici V., Egidi P., Rome, ISIME.

Statuti della provincia romana, 1930, II, éd. par Federici V., Rome, ISIME.

Statuti della Repubblica fiorentina editi a c. di Romolo Caggese-Nuova edizione, 1999, éd. par Pinto G., Salvestrini F., Zorzi A., Florence Olschki.

Statuti di Bologna dell'anno 1288, 1237, éd. par Fasoli G., Sella P., Cité du Vatican, Biblioteca Apostolica Vaticana.

Statuti di Volterra, I (1210-1224), 1251, éd. par Fiumi E., Florence, Deputazione di storia patria per la Toscana.

Statuti pistoiesi del secolo 12., 1996, éd. par Rauty N., Pistoia, Comune/Società pistoiese di storia patria.

Statuto del comune di Perugia del 1279, 1996, édité par Caprioli S., avec la collaboration de Bartoli Langel A., Cardinali C., Maiarelli A., Merli S., 2 vol., Pérouse.

Statutum Lucani communis: anno 1308, 1991, éd. par Tirelli V., Lucques, Pacini Fazzi.

Villani G., 1991, Nuova Cronica, éd. par Porta G., Parme, Guanda.

\section{Bibliographie}

Blanshei, S.R., 2010, Politics and justice in late Medieval Bologna, Leiden-Boston, Brill.

Cambiano G., 2000, Polis. Un modello per la cultura europea, Rome-Bari, Laterza. Christin 0., 2014, Vox populi. Une histoire du vote avant le suffrage universel, Paris, Seuil.

Costa, P., 1999, Civitas. Storia della cittadinanza in Europa. I. Dalla civiltà comunale al Settecento, Rome-Bari, Laterza.

Dartmann Ch., 2010, Eine Kultur der Niederlage? Wahlen in der italianischen Stadt des Hoch- un Spätmittelalters, in Dartmann Ch., G. Wassilowsky, Th. Weller (hrsg.), «Technik und Symbolik vormoderner Wahlfahren», Historische Zeitschrift, 52, Berlin, De Gruyter, p. 53-70. 
Dartmann Ch., 2012, Politische Interaktion in der italienischen Stadtkommune (XI-XIV Jahrundert), Ostfildern, Jan Thorbecke.

Fioravanti G., 1979, «Politiae Orientalium et Aegyptiorum. Alberto Magno e la Politica aristotelica », Annali della Scuola normale superiore di Pisa-Classe di Lettere e Filosofia, série III, $\mathrm{n}^{\circ} \mathrm{IX} / 1$, Pise, Scuola normale superiore, p. 195-246.

Gaudemet J., 1979, avec la collaboration de Dubois J., Duval A., Champagne J., Les élections dans l'Eglise latine des origines au Xvle siècle, Paris, Lanore.

Guidi G., 1972, «I sistemi elettorali agli uffici del Comune di Firenze nel primo Trecento: Il sorgere della elezione per squittinio (1300-1328) », Archivio Storico Italiano, 130, Florence, Olschki, p. 373-424

lanziti G., 2012, Writing History in Renaissance Italy. Leonardo Bruni and the Uses of the Past, Cambridge (Mass.), Cambridge (Ma.), Harvard University Press.

Isenmann E., 1997, Wahl, II. Ratswahl, dans Lexikon des Mittelalters, VIII/9, Munich, Lexma, coll. 1911-1912.

Judde de Larivière C., 2019, «Élection ducale, usages institutionnels et pratiques populaires: le tirage au sort dans la République de Venise », Participations, numéro hors-série : Tirage au sort et démocratie. Histoire, instruments, théories, p. 215-231.

Hayat S., Sintomer Y. (dir.), 2013, La représentation politique, numéro monographique de Raisons politiques, 50, Paris, Presses de Science Po.

Hollard V., 2010, Le rituel du vote. Les assemblées romaines du peuple, Paris, CNRS.

Keller H., 2014a, Il laboratorio politico del comune medievale, Naples, Liguori.

Keller H., 2014b, « Formes électorales et conception de la communauté dans les communes italiennes (XII $-\mathrm{xIV}^{\mathrm{e}}$ siècle) », Revue française de Science politique, LXIV/6, p. 1083-1107.

Maire Vigueur J.-C., Faini E., 2010, Il sistema politico dei comuni Italiani (secoli XII-XIV), Milan, Mondadori.

Manin B., 1995, Principes du gouvernement représentatif, Paris, Calmann-Lévy.

Milani G., 2003, L'esclusione dal comune. Conflitti e bandi politici a Bologna e in altre città italiane tra XII e XIV secolo, Rome, Istituto storico italiano per il Medioevo.

Moulin L., 1953, « Les origines religieuses des techniques électorales et délibératives modernes », Revue internationale d'histoire politique et constitutionnelle, nouvelle série, 10, p. 106-148.

Moulin L., 1958, « Sanior et maior pars. Note sur l'évolution des techniques électorales dans les Ordres religieux du $\mathrm{VI}^{\mathrm{e}}$ au $\mathrm{XII{ } ^ { \mathrm { e } }}$ siècle $»$, Revue historique de Droit français et étranger, série IV, XXXVI, p. 348-397 et 491-529.

Najemy J., 1982, Corporatism and Consensus in Florentine Electoral Politics, 1280-1400, Chapel Hill, University of North Carolina Press.

Peneau C., 2008, «Élections et pouvoirs politiques. Une introduction », dans Élections et pouvoirs politiques $d u v^{\mathrm{e}}{ }^{\mathrm{e}}$ au $X \mathrm{~V} \|^{\mathrm{e}}$ siècle, Actes du colloque réuni à 
Paris 12 du 30 novembre au 2 décembre 2006, Peneau C. (dir.), Paris, Bière, p. 13-40.

Richard 0., 2008, Élire pour contraindre : L'élection du conseil de Ratisbonne à la fin du Moyen Âge, dans Élections et pouvoirs politiques $d u V^{\mathrm{e}}{ }^{\mathrm{a}}$ au XVII ${ }^{\mathrm{e}}$ siècle, Actes du colloque réuni à Paris 12 du 30 novembre au 2 décembre 2006, Peneau C. (dir.), Paris, Bière, p. 197-212.

Rigaudiere A., 2002, «Les procureurs urbains en Auvergne, Velay et Lyonnais aux XIV et XV siècle », dans "La représentation dans la tradition du lus civile en Occident », Mélanges de l'Ecole française de Rome - Âge », 114/1, Rome, École française de Rome, p. 121-149.

Ruffini E., 1977, La ragione dei più : ricerche sulla storia del principio maggioritario, Bologne, Il Mulino.

Sintomer Y., 2009, «De Leonardo Bruni à Francesco Guicciardini : actualité de l'humanisme civique? », Raisons Politiques, 36, Paris, Presses de Sciences Po, p. 5-24.

Sintomer Y., 2011, Petite histoire de l'expérimentation démocratique : tirage au sort et politique d'Athènes à nos jours, Paris, La Découverte.

Tamba G., 1982, « Consigli elettorali degli ufficiali del comune bolognese alla fine del secolo XIII », Rassegna degli archivi di stato, $\mathrm{n}^{\circ} \mathrm{XLII}$, Ufficio centrale per $\mathrm{i}$ beni archivistici, Roma, p. 34-95

Tanzini L., 2014, A consiglio. La vita politica nell'Italia dei comuni, Rome-Bari, Laterza

Tanzini L., 2018, Représentation et décision politique dans les assemblées communales italiennes $d u$ XIII siècle, Raisons politiques, 72, Presses des Sciences Po, p. 53-70.

Théry J., 2001, Moyen Âge, in Perrineau P, Reynié D. (dir), Dictionnaire du vote, Paris, PUF, p. 667-678.

Vallerani M., 1994, « La città e le sue istituzioni. Ceti dirigenti, oligarchia e politica nella medievistica italiana del Novecento », Annali dell'istituto storico italiogermanico di Trento, 20, Bologne, Il Mulino, p. 165-230.

Vallerani M., 2011, «Comune e comuni: una dialettica non risolta », dans Sperimentazioni di governo nell'Italia centro-settentrionale nel processo storico dal primo comune alla signoria, Bologne, Bolonia university press, p. 9-34. 
Abstract-The practices and rhetoric of sortition in medieval public life (thirteenth-fourteenth centuries)

This paper focuses on the use of sortition within the political practices of the Italian city states during the central period of the late medieval blossoming. In such a context, sortition is not usually employed as such, but is rather one of the institutional tools of electoral designation, including cooptation, indirect choice, etc., with the use of the different systems depending on the political situation, the political aims, and the different offices involved. The aim of the paper is consequently to analyze the witnesses of the sources that relate to the real function of sortition in terms of its practical uses and ideological framework, and in particular to understand how, during the fourteenth century, the electoral system of most cities created a kind of turnover of officers within the circle of qualified citizens.

Keywords Medieval Italian communes, Sortition, Vote, Urban institutions, Medieval democracy

Lorenzo Tanzini est professeur associé d'Histoire médiévale à l'Université de Cagliari. Ses sujets de recherche portent sur l'histoire des institutions et de la culture politique dans l'Italie urbaine du Moyen Age, et notamment dans les villes communales, sur lesquelles il a publié, en 2014, A consiglio. La vita politica nell'Italia dei comuni (Roma, Laterza).

\section{Mots clés}

Communes italiennes, tirage au sort, vote, institutions urbaines, démocraties communales 